\title{
Comparison of polyclonal induction agents in pediatric renal transplantation
}

Brophy PD, Thomas SE, McBryde KD, Bunchman TE. Comparison of polyclonal induction agents in pediatric renal transplantation. Pediatr Transplantation 2001: 5: 174-178. (C) Munksgaard, 2001

Abstract: Collective pediatric data suggest that anti-T-cell induction therapy with polyclonal antibodies improves the outcome of both short- and long-term renal allograft survival. Polyclonal agents, including thymoglobulin (Thy), a rabbit anti-thymocyte globulin; Minnesota (horse) anti-lymphoblast globulin (ALG); and ATGAM, a horse anti-thymocyte globulin (ATG), all suppress B and T cells. While no specific T-cell subset marker exists to measure the adequacy of immunosuppression with polyclonal induction, flow cytometric analysis has been used to evaluate the suppression of CD3, CD4, and CD8 cells. Thy is currently undergoing pediatric trials at our center, and we have utilized ATG and ALG in previous pediatric induction protocols. ALG $(20 \mathrm{mg} / \mathrm{kg} /$ day $)$ and ATG (15 mg/kg/day) were administered over 10 days, whereas Thy ( $2 \mathrm{mg} / \mathrm{kg} /$ day) was given over 5 days. All inductions were accompanied by preoperative intravenous solumedrol $(10 \mathrm{mg} / \mathrm{kg})$ followed by oral prednisone $(2 \mathrm{mg} / \mathrm{kg} /$ day $)$ with taper. Preoperative $(1.5 \mathrm{mg} / \mathrm{kg} /$ day $)$ and post-operative $(2 \mathrm{mg} / \mathrm{kg} /$ day $)$ azathioprine was administered to patients receiving ALG or ATG. Mycophenolate mofetil (MMF) (1200 mg/m²/day) was given to the patients receiving Thy. Post-operative cyclosporin A (CsA) $(14 \mathrm{mg} / \mathrm{kg} /$ day) was started (for all groups) once renal function permitted (creatinine $<50 \%$ of baseline with brisk urine output) (trough goal 150-250 ng/mL via HPLC). Values for CD3, CD4, and CD8 T cells were determined by flow cytometry in $2-18$-yr-old renal transplant recipients, comparing the polyclonal induction agent utilized [Thy $(n=8)$, mean age $9.7 \pm 2.3 \mathrm{yr}$; ATG $(\mathrm{n}=13)$, mean age $10.1 \pm 4.1 \mathrm{yr}$; and ALG $(n=9)$, mean age $9.3 \pm 3.7 \mathrm{yr}$ ] over days $2-10$ post-induction. Data were expressed as the average percentage of cells remaining relative to the baseline T-cell subsets (day $1=100 \%$ ), because of the large age variation present in basal T-cell subset values. The flow cytometric data suggest that 5 days of Thy appears to give an equal or greater peripheral blood T-cell suppression by day 10 than a 10-day course of either ATG or ALG.

\author{
Patrick D. Brophy', \\ Susan E. Thomas", \\ Kevin D. McBryde ${ }^{1}$ \\ and Timothy E. Bunchman ${ }^{2}$
}

${ }^{1}$ Pediatric Nephrology and Transplantation, CS Mott Children's Hospital, University of Michigan, Ann Arbor, Michigan, USA, ${ }^{2}$ Pediatric Nephrology, Children's Hospital of Alabama, University of Alabama, Birmingham, Alabama, USA

Key words: pediatric renal transplantation polyclonal induction - T cells

Patrick Brophy, Pediatric Nephrology \& Transplantation, C. S. Mott Children's Hospital F6865-0297, 1505 Simpson Road East, University of Michigan, Ann Arbor, MI 48109-0297, USA Tel.: (734)-936-4210

Fax: (734)-763-6997

E-mail: pbrophy@umich.edu

Accepted for publication 11 October 2000
Many programs employ polyclonal immunosuppression as the standard of care for induction during pediatric kidney Tx $(1,2)$. Over the last $15 \mathrm{yr}$, the choice of polyclonal immunosuppres-

\footnotetext{
Abbreviations: ALG, Minnesota anti-lymphoblast globulin; ATGAM, horse anti-thymocyte globulin (ATG); CMV, cytomegalovirus; CsA, cyclosporin A; EBV, Epstein-Barr virus; FDA, Food and Drugs Administration; MMF, mycophenolate mofetil; Thy, thymoglobulin; Tx, transplantation.
}

sion has changed. In the 1980s and early 1990s, ALG was generally used in all institutions. In the early 1990s, ALG was discontinued and ATGAM (ATG) (UpJohn Pharmaceutical, Kalamazoo, MI, USA) became more commonly used. A product that has been used for polyclonal induction during the past few decades throughout Europe was recently introduced in the USA. This product, which was recently FDA approved, is being marketed under the name of 'thymoglobulin' (Thy) (SangStat, Palo Alto, CA, USA). 
We previously published data comparing the immunosuppressive effectiveness of ALG vs. ATG. By using flow cytometry, we demonstrated that ALG and ATG both resulted in T-cell suppression during the induction period of pediatric renal Tx (3).

Recent use of Thy as part of an induction protocol study at our institution allowed collection of flow cytometry data (4). This has enabled a comparison to be made of the immunosuppressive effect of ALG vs. ATG vs. Thy at the time of pediatric renal transplant induction.

\section{Materials and methods}

The transplant protocol in the pediatric transplant section of the University of Michigan has utilized polyclonal induction along with CsAbased immunosuppression, azathioprine, and prednisone. Since 1995 , Neoral ${ }^{\mathbb{R}}$ has been substituted for Sandimmune ${ }^{\mathbb{R}}$, and MMF has been substituted for azathioprine.

In 1998, this center became one of three pediatric centers across the country to study the use of Thy for induction in pediatrics. Part of that study was to monitor T-cell variations by the use of flow cytometry. This allowed the comparison of Thy T-cell suppression data with our previous data, which investigated the effects of ALG and ATG using the same standard flow cytometry technique. ALG $(20 \mathrm{mg} / \mathrm{kg} / \mathrm{day})$ and ATG $(15 \mathrm{mg} / \mathrm{kg} / \mathrm{day})$ were administered over 10 days. Thy $(2 \mathrm{mg} / \mathrm{kg} /$ day) was given over 5 days. Dosing of these agents was based on the literature and pharmaceutical recommendations. All inductions were accompanied by preoperative intravenous solumedrol $(10 \mathrm{mg} / \mathrm{kg})$ followed by oral prednisone $(2 \mathrm{mg} / \mathrm{kg} / \mathrm{day})$ with taper. Preoperative $(1.5 \mathrm{mg} / \mathrm{kg} / \mathrm{day})$ and post-operative $(2 \mathrm{mg} / \mathrm{kg} /$ day) azathioprine was administered to patients receiving ALG or ATG. MMF $\left(1200 \mathrm{mg} / \mathrm{m}^{2} /\right.$ day $)$ was given to the patients receiving Thy. Postoperative CsA (14 mg/kg/day) was started (for all groups) once renal function permitted (creatinine $<50 \%$ of baseline with brisk urine output) (trough goal $150-250 \mathrm{ng} / \mathrm{mL}$ via HPLC). Even though Thy was only infused for 5 days, data were collected for 10 days to allow a direct comparison to be made against ATG and ALG.

Patients (regardless of whether they received Thy, ALG or ATG), had baseline flow cytometry performed prior to transplant and daily for 10 days post-transplant. The flow cytometric analysis was performed on a FACScan flow cytometer (BectonDickinson, Immunocytometry Systems, San Jose, CA, USA) and used during all analyses, indepen- dently of era or induction agent. A murine monoclonal antibody panel to CD3, CD4, and CD8 was used for analysis. Results were reported as absolute numbers. For the benefit of comparison (as a result of the large age variation in baseline T-cell numbers, i.e. CD3 at baseline ranged from 7300 cells $/ \mathrm{mm}^{3}$ to 457 cells $/ \mathrm{mm}^{3}$ ), data were converted to percentages and represent the percentage of cells remaining relative to the baseline values. The pretransplant level (day 1) was regarded as $100 \%$ and the number of cells remaining on each subsequent day was reported as a percentage, relative to the day-1 baseline value.

Data for CD3, CD4, and CD8 suppression results (as measured by flow cytometry) were collected from 2-18-yr-old renal transplant reci-

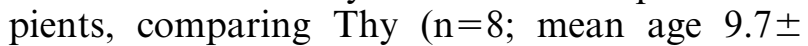
$2.3 \mathrm{yr})$, ATG $(\mathrm{n}=13$; mean age $10.1 \pm 4.1 \mathrm{yr})$, and ALG $(n=9$ mean age $9.3 \pm 3.7$ yr $)$ vs. day postinduction. To our knowledge, no patients developed infections that may have influenced the peripheral T-cell count. In addition, the patient groups were each composed of individuals who developed end-stage renal disease from diverse etiologies (i.e. congenital dysplasia/focal segmental glomerulosclerosis).

No adjustments of the polyclonal agents were made based on flow cytometry data. However, based on local standard of care, a 50\% reduction in dose of the ALG, ATG, or Thy was made if the white blood cell count was $<4000$ or the platelet count was $<100,000$. A white blood cell count of $<2000$ or a platelet count of $<50,000$ prompted withholding the dose. Missed doses were not made up. None of these patients received treatment with ALG or ATG for longer than 10 days, or with Thy for longer than 5 days, and no diminution of MMF or azathioprine dosing occured during these initial 10 days post-Tx. The average number of days of treatment with ALG was $9.1 \pm 0.9,9.7 \pm 0.3$ days with ATG, and $4.1 \pm 0.5$ days with Thy.

The Thy induction study was approved by the Institution Review Board of the University of Michigan.

\section{Results}

Table 1 illustrates data for CD3, CD4, and CD8 suppression, comparing Thy $(\mathrm{n}=8)$, ATG $(n=13)$, and ALG $(n=9)$ vs. day post-induction. Evaluation of flow cytometry data suggests that 5 days of treatment with Thy appears to give equal or greater peripheral blood T-cell suppression on day 10 than a 10-day course of either ATG or ALG. These data are represented 
Table 1. This table presents the mean averaged data (in \%) for each treatment group comparing the suppression of CD3, CD4, and CD8 cells by anti-thymocyte globulin (ATG) vs. Minnesota anti-lymphoblast globulin (ALG) vs. thymoglobulin (Thy) for days post-induction. Data are expressed as average percentage of cells remaining from baseline T-cell subsets. The pretransplanted level (day 1) was considered to be $100 \%$ and then the percentage of cells on each subsequent day was reported relative to that baseline value.

\begin{tabular}{crrrrrrrrrr}
\hline & \multicolumn{8}{c}{ Day post-induction } & \\
\cline { 2 - 8 } & 1 & 2 & 3 & 4 & 5 & 6 & 7 & 8 & 9 & 10 \\
\hline CD3 & & & & & & & & & & \\
ATG & $100 \%$ & $8 \%$ & $12 \%$ & $16 \%$ & $19 \%$ & $22 \%$ & $36 \%$ & $49 \%$ & $50 \%$ & $52 \%$ \\
ALG & $100 \%$ & $19 \%$ & $5 \%$ & $2 \%$ & $7 \%$ & $10 \%$ & $54 \%$ & $54 \%$ & $54 \%$ & $69 \%$ \\
Thy & $100 \%$ & $3 \%$ & $2 \%$ & $2 \%$ & $1 \%$ & $2 \%$ & $2 \%$ & $3 \%$ & $4 \%$ & $16 \%$ \\
CD4 & & & & & & & & & & \\
ATG & $100 \%$ & $8 \%$ & $11 \%$ & $14 \%$ & $17 \%$ & $21 \%$ & $35 \%$ & $48 \%$ & $51 \%$ & $53 \%$ \\
ALG & $100 \%$ & $18 \%$ & $6 \%$ & $1 \%$ & $5 \%$ & $8 \%$ & $44 \%$ & $21 \%$ & $32 \%$ & $73 \%$ \\
Thy & $100 \%$ & $2 \%$ & $1 \%$ & $1 \%$ & $1 \%$ & $1 \%$ & $1 \%$ & $2 \%$ & $2 \%$ & $14 \%$ \\
CD8 & & & & & & & & & & \\
ATG & $100 \%$ & $11 \%$ & $15 \%$ & $21 \%$ & $21 \%$ & $23 \%$ & $41 \%$ & $79 \%$ & $68 \%$ & $56 \%$ \\
ALG & $100 \%$ & $28 \%$ & $8 \%$ & $3 \%$ & $14 \%$ & $19 \%$ & $27 \%$ & $35 \%$ & $45 \%$ & $27 \%$ \\
Thy & $100 \%$ & $4 \%$ & $4 \%$ & $4 \%$ & $2 \%$ & $2 \%$ & $5 \%$ & $7 \%$ & $11 \%$ & $34 \%$ \\
\hline
\end{tabular}

graphically in Figs 1-3. An almost steady-state recovery of CD3 and CD4 T cells was observed in the ATG group.

Neither rejection nor infection (i.e. CMV) incidence was part of this analysis, and the analysis is complicated further because of the switch from Sandimmune to Neoral, and the transition from azathioprine to MMF.

\section{Discussion}

Previous studies have investigated the use of flow cytometry as a measure of immunosuppression for polyclonal induction. Polyclonal agents have been shown to be immunosuppressive and can be monitored by flow cytometry $(3,5,6)$. Although

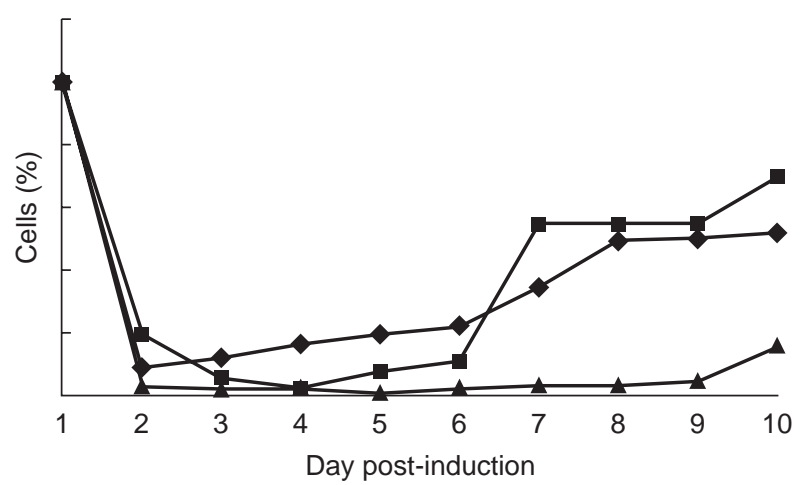

Fig. 1. The effect of thymoglobulin (Thy, $\mathbf{\Delta}$ ), antithymocyte globulin (ATG, $\bullet$ ) or Minnesota antilymphoblast globulin (ALG, - on CD3 T cells over 10 days of flow cytometric analysis. Data are expressed as the percentage of cells remaining relative to the preinduction level, represented on day 1 at $100 \%$. Note that Thy was administered for only the first 5 days while ATG and ALG were administered over the full 10 days.

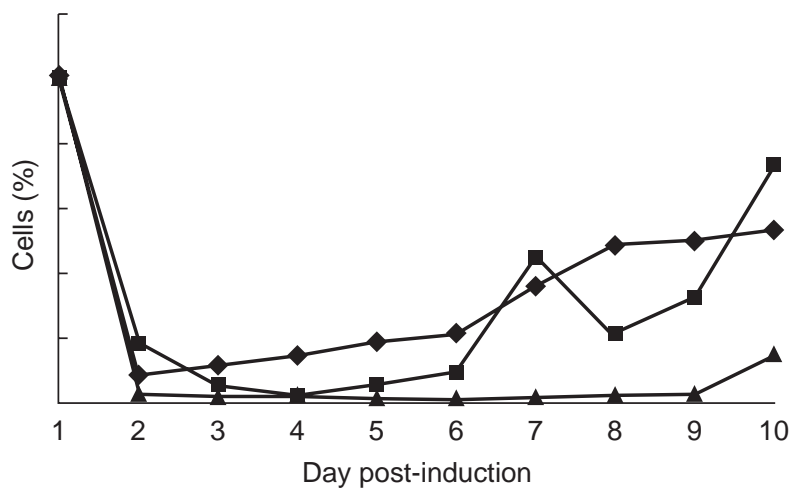

Fig. 2. The effect of thymoglobulin (Thy, $\mathbf{\Delta}$ ), antithymocyte globulin (ATG, or Minnesota antilymphoblast globulin (ALG, $\mathbf{\square}$ ) on CD4 $\mathrm{T}$ cells over 10 days of flow cytometric analysis. Data are expressed as the percentage of cells remaining relative to the preinduction level, represented on day 1 at $100 \%$. Note that Thy was administered for only the first 5 days while ATG and ALG were administered over the full 10 days.

immunosuppressive, it has been previously demonstrated that the use of polyclonal agents is not $100 \%$ protective and transplant rejection can occur during induction (7). Additionally, monitoring by flow cytometry has not been found to be a useful tool in predicting when to implement dose adjustments. This is because, in part, of the relatively slow turnaround time for obtaining results, as well as the lack of specific data demonstrating the benefit of monitoring (6, $8,9)$. There appears to be greater benefit of T-cell monitoring when monoclonal (OKT3), rather than polyclonal, agents are used; however, this too is hindered by a slow turnaround time and the lack of controlled studies which demonstrate monitoring as being absolutely effective (10).

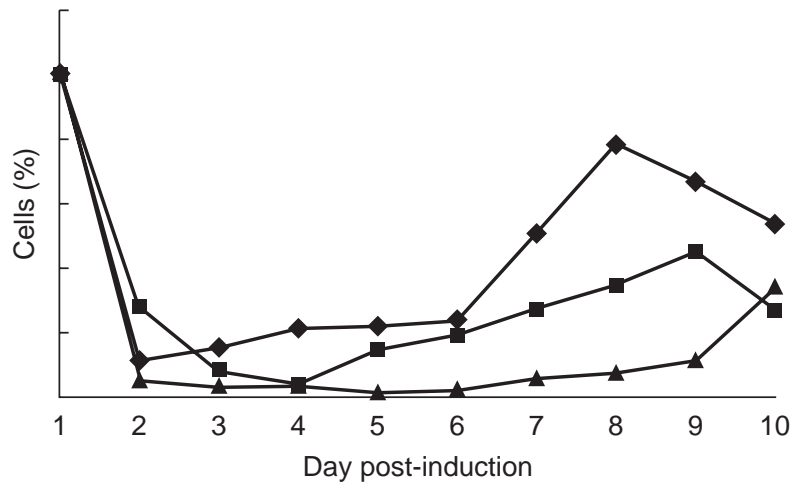

Fig. 3. The effect of thymoglobulin (Thy, $\mathbf{\Delta}$ ), antithymocyte globulin (ATG, $\diamond$ ) or Minnesota antilymphoblast globulin (ALG, - $)$ on CD8 T cells over 10 days of flow cytometric analysis. Data are expressed as the percentage of cells remaining relative to the preinduction level, represented on day 1 at $100 \%$. Note that Thy was administered for only the first 5 days while ATG and ALG were administered over the full 10 days. 
What monitoring may allow is insight into the relative effect of the T-cell suppression of the polyclonal agent. During this time-period, the children who received ALG or ATG received azathioprine and Sandimmune, while the children receiving Thy received MMF and Neoral. Data, to date, have not demonstrated that either MMF or Neoral affects T-cell cytometric analyses (11). Therefore, it would appear that these data reveal T-cell suppression specifically for each of the three different polyclonal induction agents.

The potential for antibody development to either horse- (ALG, ATG) or rabbit (Thy)-based polyclonal induction remains a concern. Filo et al. suggested that $20 \%$ of patients who receive horsebased polyclonal agents (ALG, ATG) develop anti-horse antibodies, potentially making the reuse of these drugs problematic (12). Data are being collected with respect to the potential development of anti-rabbit antibodies with the use of Thy-based induction protocols.

A major concern with utilizing this drug may be that of over-immunosuppression. Children who are at a risk of infection (i.e. with EBV or CMV) may be more susceptible during Thy-based induction protocols. Therefore, in those children who are deemed to be 'at risk', attention to infection prevention protocols and, perhaps, close monitoring of total immunosuppression in each patient may be required. Reviewing flow cytometric data for immunosuppression may be one tool employed in this monitoring system.

In European studies it has been suggested that the use of Thy may allow steroid-free protocols to be implemented (13). However, we have, as yet, had no experience with this approach, and data to date for steroid withdrawal or steroid-free protocols in pediatric patients have not been encouraging.

\section{Conclusion}

The data presented in this study suggest that a 5-day induction with Thy may produce an equal or more pronounced peripheral blood T-cell suppression at day 10 post-transplant than a 10-day course of either ATG or ALG, as measured by T-cell flow cytometry. Whether this is because Thy is a rabbit-based immunosuppression agent whereas ATG and ALG are horse-based, or because Thy is a more T-cellspecific agent, is speculative. Whilst we do not advocate utilizing flow cytometric T-cell analysis in decision-making regarding dosage adjustments during polyclonal induction, there may be a role for its use in monitoring individual patient immunosuppression levels. Additionally, continued analysis of this type of data may reveal a specific role for immunosuppression efficacy prediction using flow cytometry, similar to that which exists for the use of monoclonal agents.

One could speculate that if a program's protocol utilizes 10 days of polyclonal immunosuppression during induction then perhaps a shorter course of Thy could be used with a potential effect on length of stay. To fully evaluate the clinical effectiveness of Thy in the pediatric population, data must be collected over a longer period of time. These data must address rejection and infection incidence as well as development of lymphoproliferative disease.

\section{References}

1. Benfield MR, McDonald R, Sullivan EK, Stablein DM, TEJani A. The 1997 Annual Renal Transplantation in Children Report of the North American Pediatric Renal Transplant Cooperative Study (NAPRTCS). Pediatr Transpl 1999: 3: 152-167.

2. Ettenger RB. Special considerations in pediatric renal transplantation. In: Norman DJ, SuKI WN, eds. Primer on Transplantation. New Jersey: American Society of Transplant Physicians, 1999: 251-264.

3. Bunchman TE. Flow cytometry: its use in pediatric renal transplantation utilizing polyclonal induction. Cytometry (Comms Clin Cytometry) 1995: 22: 16-21.

4. Bartosh SM, Brophy PD, Hmiel P, Bunchman TE. Thymoglobulin induction in pediatric renal transplantation (Abstr.). First Congress of the International Pediatric Transplant Association. Venice, Italy, 2000.

5. Bell L, Girardin C, Sharma A, Goodyer P, Mazer B. Lymphocyte subsets during and after rabbit antithymocyte globulin induction in pediatric renal transplantation: sustained T cell depletion. Transplant Proc 1997: 29: 6S-9S.

6. Bourdage JS, Hamlin DM. Comparative polyclonal antithymocyte globulin and antilymphocyte/antilymphoblast globulin anti-CD antigen analysis by flow cytometry. Transplantation 1995: 59: 1194-1200.

7. Bunchman TE, Kershaw DB, Merion RM, et al. OKT3 reversal of biopsy-proven allograft rejection occurring during MALG induction in the pediatric renal recipient. Clin Transpl 1993: 7: 219-222.

8. Buchler M, Thibault G, al Najuar A, et al. Monitoring of ATG therapy by flow cytometry and lymphocyte counts in renal transplantation. Transplant Proc 1996: 28: 2817-2818.

9. Wagner FM, Tugtekin SM, Matschke K, Platzbecker U, Gulielmos V, Schuler S. Flow cytometry-controlled induction therapy with ATG and noninvasive monitoring of rejection - a modern management concept after heart transplantation. Lagenbecks Archiv fur Chirurgie 1998: Suppl. 115: 1566-1567.

10. Cinti P, Cocciolo P, Evangelista B, et al. OKT3 prophylaxis in kidney transplant recipients: drug monitoring by flow cytometry. Transplant Proc 1996: 28: 3214-3216.

11. Shanahan T. Application of flow cytometry in transplantation medicine. Immunol Invest 1997: 26: 91-101. 


\section{Brophy et al.}

12. Filo RS, Book B, Pescovitz MD, Milgrom ML, Leapman SB. Association of sensitization to horse antilymphocyte/ thymocyte globulin with recipient age and decreased renal allograft survival rates. Transplant Proc 1993: 25: 577-580.
13. Birkeland SA. Steroid-free immunosuppression after kidney transplantation with antithymocyte globulin induction and cyclosporine and mycophenolate mofetil maintenance therapy. Transplantation 1998: 66: 1207-1210. 